\section{Estimation of strabismic amblyopia by small-sized concentric gratings in preverbal children}

J Tejedor and JM Rodríguez
Conclusions The small-sized Concentric Gratings procedure improves detection and estimation of amblyopia depth in strabismus when compared with classic linear gratings. Eye (2008) 22, 1110-1116; doi:10.1038/sj.eye.6702853; published online 4 May 2007

Keywords: amblyopia; preferential looking; strabismus; esotropia

\section{Introduction}

Evaluating intensity of amblyopia in preverbal children is a challenge, especially in cases with strabismus. Preferential looking techniques estimate visual acuity in children under 3 years, but reports indicate that, by using these procedures, strabismic amblyopia may be underestimated, because grating acuity is better than recognition acuity in strabismic amblyopes. ${ }^{1-7}$

Improvement of detection and quantification of strabismic amblyopia has been attempted in several ways. For example, the characteristic deterioration of Vernier acuity (hyperacuity) in strabismic amblyopia ${ }^{3,4,8-13}$ has led to using detection of radial sinusoidal deformation of contours in preferential looking or forced choice procedures to improve the accuracy in estimation of strabismic amblyopia. ${ }^{14,15}$ We have tested the possibility of achieving this goal by a different route, that is, modifying some characteristics of the linear grating Teller Acuity Cards test usually argued as the cause of underestimating amblyopia. It is argued that uncorrected astigmatism and horizontal nystagmus can affect acuity for gratings of different orientations differentially. ${ }^{16-18}$ To circumvent this limitation, we have used concentric gratings. Cortical neurons respond
Received: 30 October 2006 Accepted in revised form: 8 April 2007 Published online: 4 May 2007
Department of Ramón y Cajal, C Colmenar,

Correspondence: J Tejedor, Ophthalmology, Cojajal Madrid 28034, Spain Fax: + 34-91-3369408 E-mail: jtejedor.hrc@ salud.madrid.org

\author{
procedure and fixation preference testing score \\ Results Concentric Gratings procedure was \\ Acuity Cards with fixation preference testing \\ score $(0.7$ and 0.49 , respectively), in the \\ preverbal group $(P=0.04)$. In treated verbal \\ children, correlation of Concentric Gratings \\ and Glasgow Acuity Cards (0.90) was stronger \\ $(P=0.06)$ than correlation of Teller Acuity \\ Cards and Glasgow Acuity Cards (0.64). In \\ untreated verbal children, correlation of \\ Concentric Gratings and Glasgow Acuity \\ Cards (0.89) was stronger $(P=0.045)$ than \\ correlation of Teller Acuity Cards and \\ Glasgow Acuity Cards (0.67).
}


best to edges (light-dark boundaries) with selective orientation, ${ }^{19}$ of which only one is stimulated by linear gratings, and in many higher-order cortical neurons, considered to be functionally altered in amblyopia, receptive fields are organized concentrically, and they respond better to circular-shaped gratings. ${ }^{20}$ Another concern with Teller Acuity Cards is the large size of gratings, placed close to the subject, which might facilitate detection by the amblyopic eye, therefore impairing estimation of amblyopia, and that accuracy of estimation is moderate when half-octave step cards are used. ${ }^{6}$ We have reduced the total size of gratings, and used 1/3 octave steps (log MAR progression), following Dobson procedure to determine visual acuity. ${ }^{21,22}$

In this study, interocular acuity difference obtained in children tested by this modified technique is compared with the classic Teller Acuity Cards vs two different tests: fixation preference testing and a recognition visual acuity test (Glasgow Acuity Cards). Fixation preference is a standard test of clinical judgement useful in preverbal children, considered more sensitive in diagnosing amblyopia than the Teller Acuity Cards in the presence of strabismus, ${ }^{23}$ but it does not provide a quantitative measurement of interocular acuity difference. The reliability of fixation preference testing is good for diagnosing amblyopia accurately. ${ }^{24,25}$ On the other hand, recognition visual acuity is regarded as the standard criterion in the diagnosis of amblyopia useful in older children. The Glasgow Acuity Cards are designed according to the principles of the Bailey-Lovie acuity test (each line contains the same number of letters and the letter size decreases in a logarithmic fashion), with four letters in each size surrounded by a box, to provide horizontal and vertical contour interaction.

\section{Methods}

\section{Subjects}

The present study adhered to the tenets of the Declaration of Helsinki, and was approved by the institutional board. Informed consent was obtained from parents of children. Forty-one preverbal children (age range: 6-30 months), referred to us with the diagnosis of esotropia, were tested for monocular visual acuity using a concentric grating preferential-looking device, and the classic Teller Acuity Cards in random order, by a masked observer. The order of eye testing was also at random. Then, an ophthalmologist, masked to the results of the previous tests, completed the examination including fixation preference testing. Data recorded were obtained at referral or about 2 months after wearing glasses, when prescribed. Anisometropia $\geqslant 1 \mathrm{D}$ of spherical equivalent or $\geqslant 1.5 \mathrm{D}$ of astigmatism in any meridian (one infant), esotropia $<10$ prism dioptres or purely accommodative (one infant), organic disease of the eye or visual pathways (one infant), or uncooperative behaviour (five infants), were considered criteria for exclusion. These criteria were directed to select patients with strabismic amblyopia, excluding anisometropic amblyopia, patients with their eyes aligned while wearing glasses or visual loss due to organic disease.

A difference in visual acuity between the two eyes was not required because the ability to discriminate different levels of interocular acuity difference was essential to the study. The study plan was to examine these infants 1-3 years later, when recognition visual acuity could be determined. Seventeen of the thirty-three children included could be tested again when they were verbal (age range: 40-48 months) after treatment of amblyopia and strabismus (recession-resection procedure in eight children, and symmetric bimedial recession in nine of them). In this subgroup follow-up exam, we used the same procedures and the Glasgow Acuity Cards test (log MAR crowded) in random order, by an observer masked to the results of the other tests. To evaluate fixation preference in the follow-up exam, the 10-prism dioptre fixation test had to be used in most of them, because deviations were smaller than 10 prism dioptres. Twenty-seven additional verbal children referred for the treatment of esotropia (age range: 39-48 months), not treated previously, underwent the same examination procedures. Exclusion criteria were identical, with one anisometropic and two uncooperative children excluded.

\section{Fixation preference testing score}

We classified fixation preference according to the following scale (adapted from Wright $e t \mathrm{al}^{24}$ ):

0 : alternates fixation or holds well fixation with the nonpreferred eye for at least $5 \mathrm{~s}$, through smooth pursuit or through a blink.

2: holds fixation briefly for 1-3s.

4: unmaintained fixation or immediate refixation to the preferred eye.

The figures used in this scale are based on the estimated amblyopia depth that is usually associated to the above findings described in fixation preference, according to Wright et al. ${ }^{24}$

\section{Description of the concentric grating apparatus}

The apparatus consisted of a large grey cardboard screen $(150 \times 75 \mathrm{~cm})$ with a rectangular opening behind which stimulus cards could be held, and two grey side panels at an angle of $45^{\circ}$ with respect to the screen. Fourteen grey cards $(25 \times 50 \mathrm{~cm})$ with one central aperture

( $3 \mathrm{~mm}$ peephole) and a circular grating on one side of the 
aperture were used (diameter of $\sim 3 \mathrm{~cm}$, to subtend $1.7^{\circ}$, (Figure 1); a set with a diameter of $5.8 \mathrm{~cm}$ was also available, but not used in the present protocol). The concentric grating target size was chosen as five times the minimal angle of resolution of the lowest visual acuity value measurable by the test $(1.5$ cycles $/ \mathrm{deg}$, which in $1.7^{\circ}$ means 2.65 cycles; that is, about five times the minimal angle of resolution, represented twice in each cycle). Michaelson contrast in the square wave gratings was $92.9 \%$ and their space average luminance was equivalent to the grey background luminance $\left(\sim 20 \mathrm{~cd} / \mathrm{m}^{2}\right)$. Edge artefact surrounding the gratings was homogeneous, whereas in linear gratings changes occur depending on whether a black or white stripe is in each side. A blank grey card with only a central aperture was also available. At $1 \mathrm{~m}$ of testing distance, the range of acuity levels of the gratings varied from 1.3 to $0 \log$ MAR (Snellen equivalents $0.05-1$ ) in $1 / 3$ octave steps.

\section{Preferential looking procedures}

During testing, the infant was held at $1 \mathrm{~m}$ from the centre of the display with one eye patched, in the concentric grating test. For the classic Teller Acuity Cards (Vistech Consultants Inc., Dayton, $\mathrm{OH}$, USA), children were tested at 55 or $84 \mathrm{~cm}$ (luminance $\sim 23 \mathrm{~cd} / \mathrm{m}^{2}$ ). For both tests, we used the Dobson procedure, in which the observer did not know the absolute spatial frequency or the location of the stripes on the card..$^{21,22}$ The observer had to decide whether the child could see the stripes, and if so, whether they were on the left or the right (by fixation, pointing or verbalization cues). The acuity recorded was the highest spatial frequency the observer judged the child could see.

In a group of five preverbal and five verbal children, we tested intraday repeatability and interday reproducibility (same observer) of the Concentric Gratings procedure. The former was achieved by taking two measurements $3 \mathrm{~h}$ apart, and the latter by repeating the measurement 3 days apart. The eyes were tested in a random order. Intraclass correlation for intraday concentric grating measurements was 0.83 (95\% confidence interval (CI): 0.54-0.96), and for interday concentric grating measurements it was 0.79 (95\% CI: 0.53-0.91).

\section{Glasgow Acuity Cards procedure}

We used a flip card format with four letters in each size surrounded by a box (Keeler Instruments Inc., Broomall, PA, USA) presented at $3 \mathrm{~m}$. The child was instructed to show the letter on a key card, which was the same as the one the examiner was pointing to. Because each line contains four letters and the letter size decreases in logarithmic progression, every letter had a score of 0.025 . The scoring system was based on $\log$ MAR (we did not subtract the $\log$ MAR score from unity, to allow comparison with the Teller Acuity Cards score). The Glasgow Acuity Cards was considered as the gold standard in verbal children.

\section{Statistical analyses}

We used the paired Student's $t$-test, repeated measures ANOVA, and repeated measures ANOVA multiple comparison of all pairs test (Tukey-Kramer test) for same group comparisons. Correlation coefficients (Spearman) were compared using the Hotelling formula for testing the difference between two dependent correlations as described by Glass and Stanley. ${ }^{26}$ For calculations, we used NCSS/PASS software (Kaysville, UT, USA).

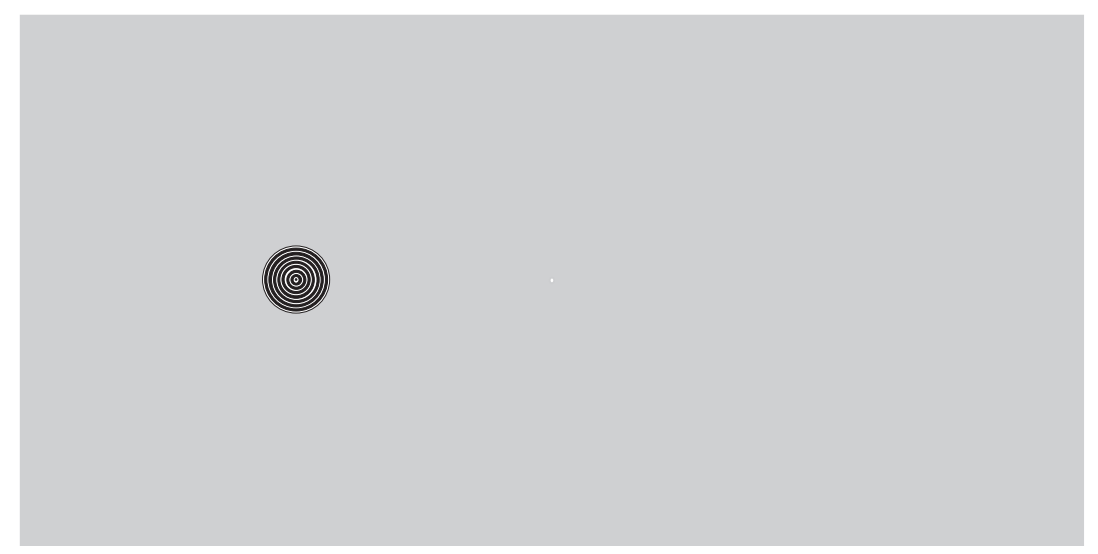

Figure 1 Sample grey card $(25 \times 50 \mathrm{~cm}$, reduced to scale) with one central aperture $(3 \mathrm{~mm}$ peephole) and a circular grating on one side of the aperture used in the concentric grating apparatus (subtending $1.6^{\circ}$ ). Michaelson contrast in the gratings is $92.9 \%$ and their space average luminance is equivalent to the grey background luminance $\left(\sim 20 \mathrm{~cd} / \mathrm{m}^{2}\right)$. The example shown illustrates a $0.2 \log$ MAR visual acuity concentric grating at $1 \mathrm{~m}$ of viewing distance (contrast is not accurately reproduced). 


\section{Results}

\section{Measurements in preverbal children}

The characteristics and variables measured in preverbal children included in the study are summarized in Table 1.

Average interocular acuity difference obtained using the Concentric Gratings procedure (0.52 log MAR, 95\% CI: 0.41-0.61) was higher than the average value obtained with the Teller Auity Cards (0.45 log MAR, 95\% CI: $0.33-0.57)$. The difference between the two measurements was statistically significant $(P=0.02$, paired $t$-test, power: $92 \%)$. Average fixation preference testing score was 2.85 (95\% CI: 2.34-3.35). The estimated correlation between Concentric Gratings interocular acuity difference and fixation preference testing score (0.7) was higher than correlation between Teller Acuity Cards interocular acuity difference and fixation preference testing score (0.49) (Table 2 and Figure 2). The difference between these two correlations was statistically significant ( $P=0.04$, Hotelling formula). Using a score of at least 2 in fixation preference testing as criterion for amblyopia, sensitivity of Teller Acuity Cards was $79.3 \%$ (23 of 29 amblyopes detected), whereas sensitivity of Concentric Gratings was $96.5 \%$ (28 of 29 amblyopes detected). Both tests had a specificity of $50 \%$.

\section{Measurements in verbal children}

Verbal treated children

Seventeen of the children examined at the preverbal age were re-examined when they were verbal, after their

Table 1 Variables measured in preverbal children before treatment

\begin{tabular}{lrrcccc}
\hline Variables & $N$ & Mean & Median & SD & Minimum & Maximum \\
\hline Age (months) & 33 & 18.54 & 19 & 8.12 & 6 & 30 \\
TAC/OD & 33 & 0.61 & 0.5 & 0.34 & 0.06 & 1.27 \\
TAC/OS & 33 & 0.56 & 0.5 & 0.31 & 0.19 & 1.27 \\
TAC/IAD & 33 & 0.45 & 0.44 & 0.33 & 0 & 1.08 \\
CG/OD & 33 & 0.61 & 0.5 & 0.35 & 0.1 & 1.3 \\
CG/OS & 33 & 0.58 & 0.6 & 0.33 & 0.1 & 1.3 \\
CG/IAD & 33 & 0.52 & 0.5 & 0.30 & 0.1 & 1.2 \\
FPS & 33 & 2.85 & 4 & 1.42 & 0 & 4 \\
Angle (PD) & 33 & 29.76 & 30 & 6.15 & 20 & 50 \\
SE/OD & 33 & 2.48 & 3 & 1.58 & -0.5 & 4.85 \\
SE/OS & 33 & 2.48 & 2.5 & 1.55 & -0.4 & 5.75 \\
\hline
\end{tabular}

Abbreviations: CG, Concentric Gratings; FPS, fixation preference testing score; IAD, Interocular Acuity Difference; PD, prism dioptres; SE, spherical equivalent in dioptres; TAC, Teller Acuity Cards.

Visual acuity is expressed in $\log$ MAR units in TAC and CG; the table was produced with NCSS. amblyopia and strabismus had been treated. The characteristics of these children at re-examination are summarized in Table 3

Average interocular acuity differences obtained using the Concentric Gratings procedure, Glasgow Acuity Cards, and Teller Acuity Cards were, respectively, $0.23 \log$ MAR (95\% CI: 0.14-0.31), $0.22 \log$ MAR (95\% CI: 0.14-0.29), and 0.19 log MAR (95\% CI: 0.11-0.27). When compared by repeated measures ANOVA, these average values were different at the limit of significance $(P=0.05)$. In a repeated measures ANOVA multiple comparison of all pairs (Tukey-Kramer test), the result obtained with Teller Acuity Cards was significantly lower than that obtained with Concentric Gratings

Table 2 Correlations between variables measured

\begin{tabular}{|c|c|c|c|}
\hline & CG IAD & TAC IAD & $\begin{array}{l}P \text { (comparison by } \\
\text { Hotelling formula) }\end{array}$ \\
\hline \multicolumn{4}{|c|}{ Pre-verbal $(n=33)$} \\
\hline FPS & 0.7 & 0.49 & 0.04 \\
\hline \multicolumn{4}{|c|}{ Verbal treated $(n=17)$} \\
\hline GAC IAD & 0.90 & 0.64 & 0.06 \\
\hline FPS & 0.81 & 0.48 & 0.05 \\
\hline \multicolumn{4}{|c|}{ Verbal non-treated $(n=24)$} \\
\hline GAC IAD & 0.89 & 0.67 & 0.045 \\
\hline FPS & 0.76 & 0.48 & 0.04 \\
\hline
\end{tabular}

Abbreviations: CG, Concentric Gratings; FPS, fixation preference testing score; GAC, Glasgow Acuity Cards; IAD, Interocular Acuity Difference; TAC, Teller Acuity Cards.

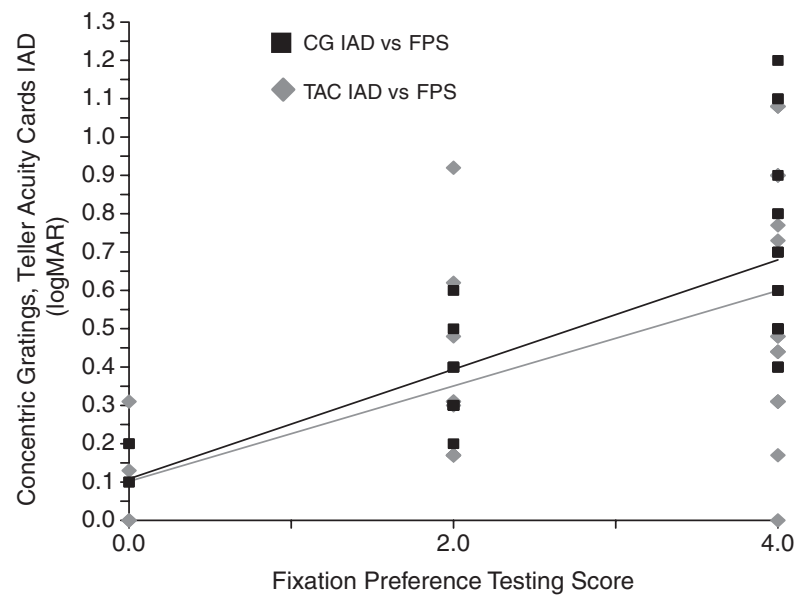

Figure 2 Scatterplot and separate regression lines for relation of Fixation Preference Testing Score with Concentric Gratings (black squares, $r=0.7$ ), and Teller Acuity Cards (grey diamonds, $r=0.49)$ interocular acuity difference in preverbal children. Difference between the two correlations is significant $(P=0.04$, Hotelling formula). 
$(P=0.04)$ and Glasgow Acuity Cards $(P=0.04)$, but the latter two tests were not considered different $(P=0.3)$. Average fixation preference testing score was $2.4(95 \% \mathrm{CI}$ : 1.44-3.35). Using a difference of at least $2 \log$ MAR lines in Glasgow Acuity Cards as criterion for amblyopia, sensitivity of Teller Acuity Cards was 54.5\% (six of 11 amblyopes detected), whereas sensitivity of Concentric Gratings was $90.9 \%$ (10 of 11 amblyopes detected). Specificity was $66.6 \%$ for the two tests.

The correlations between variables measured in estimated correlation between interocular acuity difference measured with Concentric Gratings and Glasgow Acuity Cards was stronger than that measured with Teller Acuity Cards and Glasgow Acuity Cards. The difference between these two correlations was marginally significant ( $P=0.06$, Hotelling formula). The Concentric Gratings procedure (interocular acuity difference) was also more strongly correlated with fixation preference testing score than Teller Acuity Cards $(P=0.05$, Hotelling formula). The correlation between fixation preference testing score and Glasgow Acuity Cards (interocular acuity difference) was 0.87 (95\% CI: 0.64-0.95).

\section{Verbal nontreated children}

The characteristics of verbal children included in the study, not previously treated for strabismus or amblyopia, are listed in Table 4.

Average interocular acuity differences obtained using the Concentric Gratings procedure, Glasgow Acuity Cards, and Teller Acuity Cards were, respectively, $0.36 \log$ MAR (95\% CI: 0.28-0.44), $0.39 \log$ MAR (95\% CI: treated verbal children are shown in Table 2. The

0.31-0.47), and 0.30 log MAR (95\% CI: 0.23-0.39). When compared by repeated measures ANOVA, these average values were significantly different $(P=0.002)$. In a repeated measures ANOVA multiple comparison of all pairs (Tukey-Kramer test), the result obtained with Teller Acuity Cards was lower than that obtained with Concentric Gratings ( $P=0.045$, marginal significance), and Glasgow Acuity Cards $(P=0.02)$, whereas the latter two measurements were not found to be different $(P=0.2)$. Average fixation preference testing score was 2.9 (95\% CI: 2.31-3.50). Using a difference of at least $2 \log$ MAR lines in Glasgow Acuity Cards as criterion for amblyopia, sensitivity of Teller Acuity Cards was $57.1 \%$ (12 of 21 amblyopes detected), and that of Concentric Gratings was 90.4\% (19 of 21 amblyopes detected). Specificity of the two tests was 66.6 and $83.3 \%$, respectively.

The correlations between variables measured in nontreated verbal children are listed in Table 2 . The estimated correlation between interocular acuity difference measured with Concentric Gratings and Glasgow Acuity Cards was stronger than that between Teller Acuity Cards and Glasrgow Acuity Cards. The difference between these two correlations reached significance ( $P=0.045$, Hotelling formula; Figure 3). The Concentric Gratings procedure (interocular acuity difference) was also more strongly correlated with fixation preference testing score than Teller Acuity Cards ( $P=0.04$, Hotelling formula). The correlation between fixation preference testing score and Glasgow Acuity Cards (interocular acuity difference) was 0.72 .

Table 4 Variables measured in verbal nontreated children

Table 3 Variables measured in verbal treated children

\begin{tabular}{lcccccc}
\hline Variables & $\mathrm{N}$ & Mean & Median & SD & Minimum & Maximum \\
\hline Age (months) & 17 & 40.8 & 40 & 4.07 & 36 & 47 \\
TAC/OD & 17 & 0.16 & 0.19 & 0.10 & 0.06 & 0.36 \\
TAC/OS & 17 & 0.22 & 0.19 & 0.17 & 0.06 & 0.49 \\
TAC/IAD & 17 & 0.19 & 0.13 & 0.15 & 0 & 0.43 \\
CG/OD & 17 & 0.12 & 0.1 & 0.13 & 0 & 0.4 \\
CG/OS & 17 & 0.21 & 0.2 & 0.18 & 0 & 0.5 \\
CG/IAD & 17 & 0.23 & 0.2 & 0.16 & 0 & 0.5 \\
FPS & 17 & 2.4 & 2 & 1.72 & 0 & 4 \\
GAC/OD & 17 & 0.19 & 0.12 & 0.12 & 0.1 & 0.45 \\
GAC/OS & 17 & 0.29 & 0.2 & 0.17 & 0.1 & 0.6 \\
GAC/IAD & 17 & 0.22 & 0.27 & 0.14 & 0 & 0.4 \\
Angle (PD) & 17 & 6.4 & 8 & 4.85 & -2 & 16 \\
SE/OD & 17 & 1.68 & 1.25 & 1.97 & -1.25 & 5.25 \\
SE/OS & 17 & 1.93 & 1 & 2.27 & -1 & 6.25 \\
\hline
\end{tabular}

Abbreviations: CG, Concentric Gratings; FPS, fixation preference testing score; GAC, Glasgow Acuity Cards; IAD, Interocular Acuity Difference; $\mathrm{PD}$, prism dioptres; $\mathrm{SE}$, spherical equivalent in dioptres; $\mathrm{TAC}$, Teller Acuity Cards.

Visual acuity is expressed in $\log$ MAR units in TAC, CG, and GAC; the table was produced with NCSS.

\begin{tabular}{lrrrrcc}
\hline Variables & $\mathrm{N}$ & Mean & Median & SD & Minimum & Maximum \\
\hline Age (months) & 24 & 43.27 & 42.5 & 3.33 & 39 & 48 \\
TAC/OD & 24 & 0.27 & 0.19 & 0.20 & 0.06 & 0.8 \\
TAC/OS & 24 & 0.31 & 0.275 & 0.24 & 0.06 & 0.8 \\
TAC/IAD & 24 & 0.30 & 0.305 & 0.19 & 0 & 0.61 \\
CG/OD & 24 & 0.24 & 0.15 & 0.23 & 0 & 0.9 \\
CG/OS & 24 & 0.32 & 0.3 & 0.26 & 0 & 0.9 \\
CG/IAD & 24 & 0.36 & 0.3 & 0.19 & 0 & 0.7 \\
FPS & 24 & 2.91 & 4 & 1.34 & 0 & 4 \\
GAC/OD & 24 & 0.26 & 0.135 & 0.24 & 0.05 & 0.9 \\
GAC/OS & 24 & 0.37 & 0.35 & 0.25 & 0.05 & 0.92 \\
GAC/IAD & 24 & 0.39 & 0.34 & 0.19 & 0 & 0.78 \\
Angle (PD) & 24 & 30.91 & 30 & 6.66 & 20 & 45 \\
SE/OD & 24 & 1.55 & 1.175 & 1.41 & -0.37 & 5 \\
SE/OS & 24 & 1.72 & 1.935 & 1.11 & 0 & 3.75 \\
\hline
\end{tabular}

Abbreviations: CG, Concentric Gratings; FPS, fixation preference testing score; GAC, Glasgow Acuity Cards; IAD, Interocular Acuity Difference; $\mathrm{PD}$, prism dioptres; SE, spherical equivalent in dioptres; TAC, Teller Acuity Cards.

Visual acuity is expressed in $\log$ MAR units in TAC, CG, and GAC; the table was produced with NCSS. 


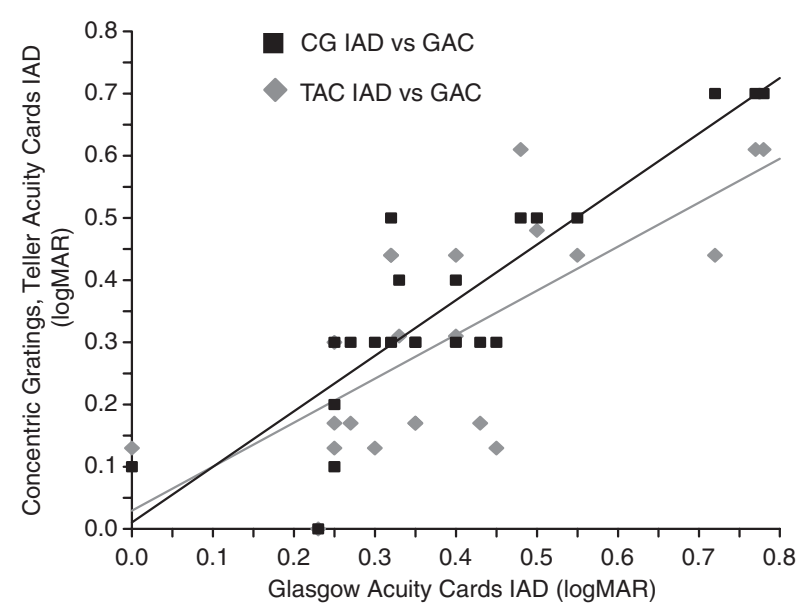

Figure 3 Scatterplot and separate regression lines for relation of Glasgow Acuity Cards interocular acuity difference with Concentric Gratings (black squares, $r=0.89$ ), and Teller Acuity Cards (grey diamonds, $r=0.67$ ) interocular acuity difference in verbal non-treated children. Difference between the two correlations is significant ( $P=0.045$, Hotelling formula).

\section{Discussion}

The use of a preferential looking small-sized concentric grating test in preverbal children improves in some degree estimation and detection of strabismic amblyopia. A direct comparison with a recognition visual acuity test cannot be made at the preverbal age. Fixation preference testing is reliable ${ }^{24}$ and more sensitive than Teller Acuity Cards in diagnosing strabismic amblyopia, ${ }^{23}$ which is usually underestimated by preferential looking, ${ }^{1-7}$ particularly in cases with greater interocular acuity difference. ${ }^{5}$ The main reason to test a different preferential looking design is that fixation preference does not provide a quantitative measurement of interocular acuity difference as recognition visual acuity does. The fixation preference testing score recorded is significantly better correlated with the Concentric Gratings than with the Teller Acuity Cards procedure in preverbal children (Figure 2). Similarly, the Glasgow Acuity Cards is better correlated with Concentric Gratings than with Teller Acuity Cards in verbal children (Figure 3).

It may be argued that the difference in average interocular acuity difference obtained with Concentric Gratings and Teller Acuity Cards cannot be considered of clinical significance, although statistical significance was reached (in part, due to the paired design and the high test-retest intraclass correlations). Yet, differences between the two methods are not required when two dependent correlations are compared, because this comparison elucidates which of the two methods correlates better with the gold standard, although they do not differ significantly, ${ }^{26,27}$ and therefore provides a better assessment of strabismic amblyopia. Using a $2 \log$ MAR lines interocular acuity difference restrictive criterion to define amblyopia, sensitivity of the Concentric Gratings is above $90 \%$ at preverbal and verbal age, whereas specificity ranges between 50 and $83 \%$. Concentric Gratings detect a higher percentage of strabismic amblyopes, as diagnosed by the gold standard tests than Teller Acuity Cards. It also improves identification of nonamblyopic children compared with classic linear gratings.

Measuring verbal children after treating amblyopia is of interest to compare tests under conditions of mild amblyopia (which adds to comparisons in preverbal and verbal untreated children with deeper amblyopia). In verbal children, it is more difficult to hold their attention, or they can move or obtain additional cues about the location of the gratings in preferential looking procedures.

Improvement in assessing strabismic amblyopia by the present grating visual acuity design may be attributed to several reasons. A reduction in target size (which impairs detection and results in a more foveal task), elimination of linear orientation preference (which influences perception differentially depending on pre-existing astigmatism or nystagmus), and finer gradation of steps, might explain in part this finding. The physiological basis of the improvement may also be related to the existence of specific cortical cells, which respond to concentric grating stimulus. The main affected site in strabismic amblyopia is the primary visual cortex, but V1 deficits alone cannot explain for impaired higher-order visual perceptual tasks observed in amblyopia. A great percentage of cells tested in macaque area V4, an intermediate stage of the visual hierarchy, respond significantly more to non-Cartesian than to Cartesian gratings. Among cells selective for non-Cartesian gratings, those that prefer concentric gratings are most common. Cells selective for non-Cartesian gratings may represent an important intermediate stage in pattern recognition. ${ }^{19}$ A functional magnetic resonance imaging study demonstrated that the human area V4 is more strongly activated by concentric and radial patterns than by conventional sinusoidal gratings. ${ }^{28} \mathrm{~A}$ recent report indicates that radial frequency concentric gratings stimuli are processed by a more foveal-dependent brain system operating at high luminance and contrast levels, unlike sine-wave gratings, which are processed by one working at low luminance and contrast levels. ${ }^{29}$

A limitation of the present study is the relatively small size of samples, particularly in the previously treated verbal children. In this group, failure to complete followup and lengthening of the study caused losses. However, significance was reached in one of the two comparisons 
of dependent correlations reported in this group. In the two other groups, the power of the tests used was good with the number of subjects included. Recruitment of children with the required characteristics is less frequent than we guessed initially in a paediatric ophthalmology clinic.

The current study demonstrates enhanced assessment of strabismic amblyopia by the Concentric Gratings procedure (in which influence of several introduced changes is of notice) relative to classic linear preferential looking cards. It is conceivable that using some other different approaches in modifying the characteristics of preferential looking grating visual acuity tests, might further improve the ability to evaluate strabismic amblyopia in preverbal children. Additional studies are needed to corroborate this hypothesis.

\section{Acknowledgements}

The authors wish to acknowledge Víctor Abraira for his help in statistics.

\section{References}

1 Gstalder RJ, Green DG. Laser interferometric acuity in amblyopia. J Pediatr Ophthalmol 1971; 8: 251-256.

2 Hess RF, Campbell FW, Greenhalgh T. On the nature of the neural abnormality in human amblyopia: neural aberrations and neural sensitivity loss. Pflugers Arch 1978; 377: 201-207.

3 Levi DM, Klein SA. Hyperacuity and amblyopia. Nature 1982; 298: 268-270.

4 Levi DM, Klein SA. Differences in Vernier discrimination for gratings between strabismic and anisometropic amblyopes. Invest Ophthalmol Vis Sci 1982; 23: 398-407.

5 Mayer DL, Fulton AB, Rodier D. Grating and recognition acuities of pediatric patients. Ophthalmology 1984; 91: 947-953.

6 Mayer DL. Acuity of amblyopic children for small field gratings and recognition stimuli. Invest Ophthalmol Vis Sci 1986; 27: 1148-1153.

7 Selenow A, Ciuffreda KJ, Mozlin R, Rumpf D. Prognostic value of laser interferometric visual acuity in amblyopia therapy. Invest Ophthalmol Vis Sci 1986; 27: 273-277.

8 Bedell HE, Flom MC. Monocular spatial distorsion in strabismic amblyopia. Invest Ophthalmol Vis Sci 1981; 20: 26-28.

9 Bedell HE, Flom MC. Normal and abnormal space perception. Am J Optom Physiol Opt 1983; 60: 426-435.

10 Levi DM, Klein SA. Vernier acuity, crowding, and amblyopia. Vision Res 1985; 25: 979-991.

11 McKee SP, Schor CM, Steinman SB, Wilson N, Koch GG, Davis SM et al. The classification of amblyopia on the basis of visual and oculomotor performance. Trans Am Ophthalmol Soc 1992; 90: 123-148.

12 Kiorpes L, Movshon JA. Amblyopia: a developmental disorder of the central visual pathways. Cold Spring Harbor Symposium on Quantitative Biology 1996; 61: 39-48.

13 Carkeet A, Levi DM, Manny RE. Development of Vernier acuity in childhood. Optom Vis Sci 1997; 74: 741-750.

14 Hess RF, Wang YZ, Demanins R, Wilkinson F, Wilson HR. A deficit in strabismic amblyopia for global shape detection. Vision Res 1999; 39: 901-914.

15 Birch EE, Swanson WH, Wang Y-Z. Infant hyperacuity for radial deformation. Invest Ophthalmol Vis Sci 2000; 41: 3410-3440.

16 Teller DY, Allen JL, Regal DM, Mayer DL. Astigmatism and acuity in two primate infants. Invest Ophthalmol Vis Sci 1978; 17: 344-349.

17 Gwiazda J, Mohindra I, Brill S, Held R. Infant astigmatism and meridional amblyopia. Vision Res 1985; 25: 1269-1276.

18 Dobson V. Visual acuity testing in infants: from laboratory to clinic. In: Simons K (ed). Early Visual Development, Normal and Abnormal. Oxford University Press: Oxford, New York, 1993; 318-334.

19 Hubel DH, Wiesel TN. Receptive fields, binocular interaction and functional architecture in the cat's visual cortex. J Physiol (London) 1962; 160: 106-154.

20 Gallant JL, Braun J, Van Essen DC. Selectivity for polar, hyperbolic, and cartesian gratings in macaque visual cortex. Science 1993; 259: 100-103.

21 Dobson V, Carpenter NA, Bonvalot K, Bossler J. The acuity card procedure: interobserver agreement in infants with perinatal complications. Clin Vis Sci 1990; 6: 39-48.

22 Lewis TL, Reed MJ, Maurer D, Wyngaarden PA, Brent HP. An evaluation of acuity card procedures. Clin Vis Sci 1993; 8: 591-602.

23 Ellis GS, Hartmann EE, Love A, May JG, Morgan KS. Teller Acuity Cards versus clinical judgment in the diagnosis of amblyopia with strabismus. Ophthalmology 1988; 95: 788-791.

24 Wright KW, Edelman PM, Walonker F, Yiu S. Reliability of fixation preference testing in diagnosing amblyopia. Arch Ophthalmol 1986; 104: 549-553.

25 Wright KW, Walonker F, Edelman P. 10-Diopter fixation test for amblyopia. Arch Ophthalmol 1981; 99: 1242-1246.

26 Glass GV, Stanley JC. Statistical methods in education and psychology. Upper Saddle River. Prentice-Hall: Englewood Cliffs, NJ, 1970; 1: 310-311.

27 Nesselroad JM, Flacco VA, Phillips DM, Kruse J. Accuracy of automated finger blood pressure devices. Fam Med 1996; 28: 189-192.

28 Wilkinson F, James TW, Wilson HR, Gati JS, Menon RS, Goodale MA. An fMRI study of the selective activation of human extrastriate form vision areas by radial and concentric gratings. Curr Biol 2000; 10: 1455-1458.

29 Simas ML, Nogueira RM, Santos NA. Radial frequency stimuli and sine-wave gratings seem to be processed by distinct contrast brain mechanisms. Braz J Med Biol Res 2005; 38: $419-430$. 